\title{
A!
}

This is an electronic reprint of the original article.

This reprint may differ from the original in pagination and typographic detail.

Pöykkö, Sami; Puska, M.J.; Nieminen, R.M.

\section{Metastability of the antistructure pair in GaAs}

Published in:

Physical Review B

DOI:

10.1103/PhysRevB.55.6914

Published: 15/03/1997

Document Version

Publisher's PDF, also known as Version of record

Please cite the original version:

Pöykkö, S., Puska, M. J., \& Nieminen, R. M. (1997). Metastability of the antistructure pair in GaAs. Physical Review B, 55(11), 6914-6917. https://doi.org/10.1103/PhysRevB.55.6914

This material is protected by copyright and other intellectual property rights, and duplication or sale of all or part of any of the repository collections is not permitted, except that material may be duplicated by you for your research use or educational purposes in electronic or print form. You must obtain permission for any other use. Electronic or print copies may not be offered, whether for sale or otherwise to anyone who is not an authorised user. 


\title{
Metastability of the antistructure pair in GaAs
}

\author{
S. Pöykkö, M. J. Puska, and R. M. Nieminen \\ Laboratory of Physics, Helsinki University of Technology, FIN-02150 Espoo, Finland
}

(Received 29 October 1996)

\begin{abstract}
We have studied the metastability of the antistructure (arsenic-antisite gallium-antisite) pair in GaAs using self-consistent, parameter-free total energy methods. Our calculations predict that this defect complex exhibits metastability similar to that of the isolated arsenic antisite. However, the antistructure pair has ionization levels in the band gap in the metastable configuration, unlike the isolated arsenic antisite. The ionization levels enable absorption of infrared light in the metastable state. The results are used to discuss and interpret the arsenicantisite-type defects observed experimentally in electron-irradiated GaAs. [S0163-1829(97)04011-3]
\end{abstract}

The most important native point defect in undoped GaAs crystals grown under the As-rich conditions is the so-called EL2 defect. The EL2 defect shows interesting and important metastability. ${ }^{1}$ A key feature of the metastable state of the EL2 defect is that it has been found optically and electrically inactive. In most of the microscopic models for the EL2 defect the arsenic antisite $\left(\mathrm{As}_{\mathrm{Ga}}\right)$ is at least a constituent of the defect, but the identification of the EL2 as an isolated arsenic antisite is still somewhat controversial. ${ }^{2}$

The introduction of As-antisite-related defects in electron ${ }^{3}$ and neutron ${ }^{4}$ irradiation as well as during plastic deformation $^{5}$ has been actively studied. Recently, three different electron-irradiation-induced $\mathrm{As}_{\mathrm{Ga}}$-related defects possessing metastability have been observed by magnetic circular dichroism of absorption (MCDA). ${ }^{6}$ One of these is obtained after subsequent thermal annealing at room temperature. This defect has been studied with the optically detected electron nuclear double resonance (ODENDOR) technique and suggested to be an antistructure pair, which is an $\mathrm{As}_{\mathrm{Ga}}$ defect with a Ga-antisite $\left(\mathrm{Ga}_{\mathrm{As}}\right)$ defect located in the next-nearest As shell of the As antisite. ${ }^{7}$ The structure is given schematically in Fig. 1. Information on the atomic structure of the metastable state of this defect has been obtained by positron lifetime measurements. ${ }^{8}$ These experiments indicate that the defect has an associated vacancy in its metastable state. The defect seen in the positron lifetime measurements is associated with that detected in MCDA, i.e., to the antistructure pair. ${ }^{9}$ The concentration of these irradiation-induced metastable defects may be much higher

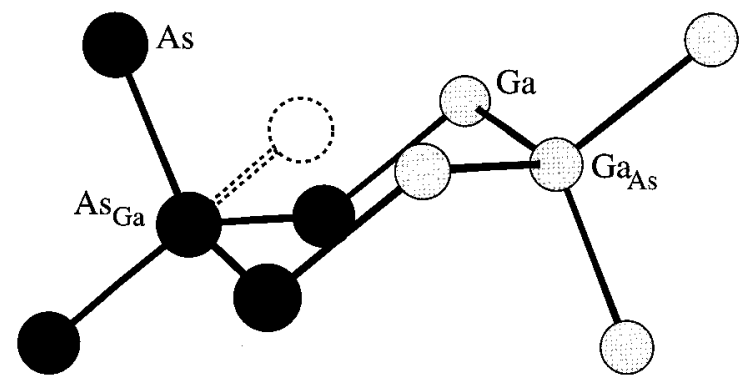

FIG. 1. Schematic view of the next-nearest-neighbor antistructure pair in GaAs. The metastable interstitial position of the $\mathrm{As}_{\mathrm{Ga}}$ antisite is shown by dashed lines. than the concentration of the native EL2 defect. ${ }^{8}$

Some properties of the irradiation-induced $\mathrm{As}_{\mathrm{Ga}}$-related defects differ crucially from those of the native $\mathrm{As}_{\mathrm{Ga}}$-related defects. For example, the existence of the metastability has not always been detected. The main difference between the EL2 defect and the irradiation-induced antistructure defect is that the latter absorbs infrared light in the metastable configuration, suggesting that it has an ionization level in the band gap in the metastable configuration. ${ }^{9}$ The stable state of the irradiation-induced $\mathrm{As}_{\mathrm{Ga}}$ defect can be optically recovered with 0.85 or $1.3-\mathrm{eV}$ photons at $20 \mathrm{~K}$, while this is not possible for the native EL2 defect. ${ }^{6,9}$ Another interesting difference is that the metastable state of the irradiationinduced defect may be paramagnetic. ${ }^{6}$

In this paper we report studies of the antistructure pair using first-principles electronic-structure methods. ${ }^{10}$ Our goal is to show that the calculated properties of the antistructure pair indeed explain the main experimental properties of the above-mentioned irradiation-induced metastable defect. Our calculations are based on the density-functional theory where the electron exchange-correlation energy is calculated within the local-density approximation (LDA). ${ }^{11} \mathrm{We}$ use first-principles norm-conserving pseudopotentials ${ }^{12}$ for both $\mathrm{Ga}$ and As. For the plane-wave basis set a high cutoff energy of 15 Ry has been used to ensure accurate results. Calculations are performed with the supercells containing 32 or 64 atoms. The Brillouin zone sampling consists of the $2 \times 2 \times 2$ Monkhorst-Pack ${ }^{13} \mathbf{k}$-point mesh for the 32 -atomsite supercell, and for the 64-atom-site supercell the $2 \times 2 \times 2$ Chadi-Cohen ${ }^{14} \mathbf{k}$-point mesh has been employed. Further computational details can be found in Ref. 15.

According to the calculations the charge states of the $\mathrm{As}_{\mathrm{Ga}}$ and $\mathrm{Ga}_{\mathrm{As}}$ antisites are over a wide region for the electron chemical potential $2+$ and $2-$, respectively (see Fig. 2 ). Within these kinds of conditions the antisites are expected to attract each other via the Coulomb interaction. We have studied the strength of the interaction by calculating the total energy of the antisite pair for the nearest-, next-nearest-, and third-nearest-neighbor configurations. Energetically the most favorable is the nearest-neighbor antistructure pair. The binding energies obtained for the nearest-, next-nearest, and third-nearest-neighbor antistructure pairs in the 2- charge state are $1.2,0.4 \mathrm{eV}$, and $0.3 \mathrm{eV}$, respectively. However, the nearest-neighbor antisite pair does not have ionization levels 
CBM

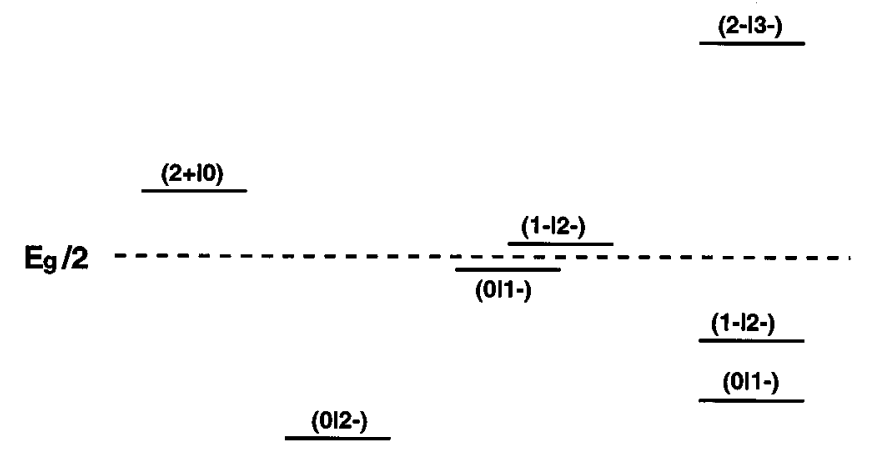

VBM

\section{$\begin{array}{llll}A s_{\mathrm{Ga}} & \mathrm{Ga}_{\mathrm{As}} & \mathrm{As}_{\mathrm{Ga}} \mathrm{Ga}_{\mathrm{As}} & \mathrm{V}_{\mathrm{Ga}} \mathrm{As}_{i} \mathrm{Ga}_{\mathrm{As}}\end{array}$}

FIG. 2. Positions of the ionization levels in the band gap. The results for the isolated $\mathrm{As}_{\mathrm{Ga}}$ and $\mathrm{Ga}_{\mathrm{As}}$ antisites as well as for the next-nearest-neighbor antistructure defect in the substitutional $\left(\mathrm{As}_{\mathrm{Ga}} \mathrm{Ga}_{\mathrm{As}}\right)$ and interstitial $\left(V_{\mathrm{Ga}} \mathrm{As}_{i} \mathrm{Ga}_{\mathrm{As}}\right)$ configurations are shown. The level positions for the isolated antisites are in agreement with those given in Ref. 16.

in the band gap, and thus the nearest-neighbor pair cannot effectively absorb infrared light in the metastable state. Therefore it cannot be the defect seen in the experiments of Kuisma et al. ${ }^{9}$ Also, the nearest-neighbor structure does not agree with the analysis of the ODENDOR lines. ${ }^{7}$ Moreover, recent molecular-dynamics simulations ${ }^{18}$ for defect formation under electron irradiation have shown that although the open and closed [111] directions are clearly the most probable ones for antisite formation, the directions towards to the second nearest neighbor are also favored in this sense. Therefore, the creation of the next-nearest-neighbor antistructure pairs is likely in electron irradiation succeeded by annealing. A low-energy barrier for the recovery of the nearest-neighbor antistructure pair could explain the nonexistence of the nearest-neighbor defect after annealing. Unfortunately, we are not able to estimate this energy barrier. Therefore we have omitted more detailed studies of this configuration.

As the distance between the two antisites increases, they become decoupled and the arsenic antisite becomes like an isolated one, which cannot explain the experimental findings either. For these reasons in the following we consider only the next-nearest-neighbor antistructure pair and call it "the anti-structure defect' for short.

The antistructure defect has neutral and negative charge states $(0,1-, 2-, 3-)$. In principle also a $4-$ charge state is possible for the antistructure defect, but it turns out that it is not the lowest-energy charge state for any value of the electron chemical potential (see the ionization levels in Fig 2 ). In the neutral state the highest occupied electron states are in the band gap close to the top of the valence band. They are related to the deep levels induced by an isolated $\mathrm{Ga}_{\mathrm{As}}$ antisite. In the doubly negative charge state the highest states are related to those of an isolated $\mathrm{As}_{\mathrm{Ga}}$ antisite. Our totalenergy calculations show that the defect has a metastable configuration similar to the large-lattice relaxation ${ }^{17}$ (LLR) model for the EL2 defect: The metastable state is achieved when the arsenic antisite moves from the Ga site to the open

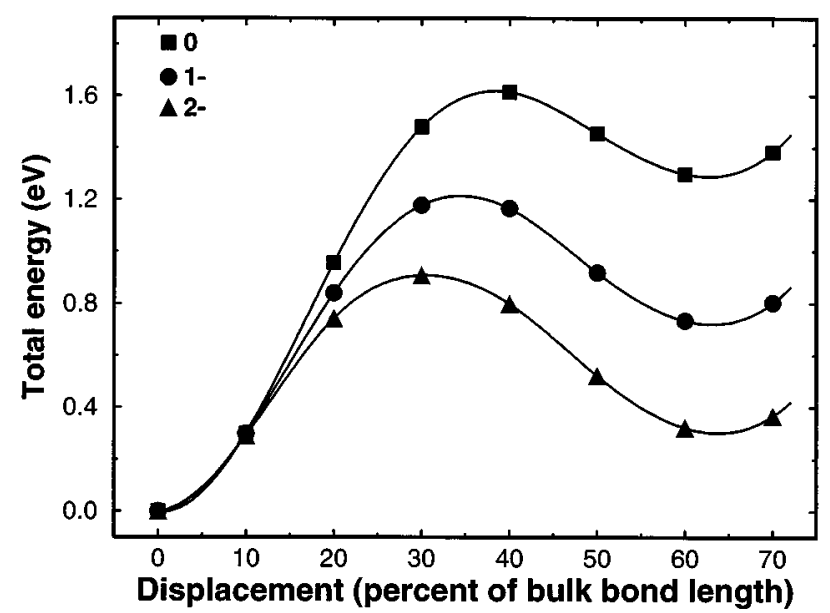

FIG. 3. Total energy of the antistructure defect as a function of the As-ion displacement from the Ga substitutional position along the open [111] direction. The distance between the antisites decreases with increasing displacement. The zero of the energy corresponds to the total-energy in the substitutional ionic configuration. No other ionic relaxations have been allowed. The three curves correspond to different charge states $(0,1-, 2-)$. The local minima in the total energy curves at $\sim 60 \%$ displacement indicate the existence of metastable ionic configurations.

[111] direction by $\sim 60 \%$ of the bulk bond length (see Fig. 1). A vacancy in the metastable state of the antistructure defect is thus created. The presence of a vacancy in the metastable state of the antistructure defect is also directly probed by positron lifetime measurements. ${ }^{8}$ There are three inequivalent open [111] directions for the movement of the $\mathrm{As}_{\mathrm{Ga}}$ antisite in the case of the antistructure defect. The movement of the $\mathrm{As}_{\mathrm{Ga}}$ antisite in the [111] direction in which the distance between the $\mathrm{As}_{\mathrm{Ga}}$ and $\mathrm{Ga}_{\mathrm{As}}$ antisites decreases is favored. This is because the defect pair gains in the Coulomb attraction. The evaluation of the total energy, when the As ion is displaced in this direction from the substitutional $\mathrm{Ga}$ site is shown in Fig. 3 for the charge states $0,1-$, and $2-$. The metastability exists for all of these charge states. In comparison, the isolated $\mathrm{As}_{\mathrm{Ga}}$ antisite exhibits metastability only in the neutral charge state when the deep levels in the band gap are occupied with two electrons. ${ }^{17}$ The corresponding charge state of the antistructure defect is $2-$. Indeed the curve for the charge state $2-$ in Fig. 3 resembles very closely that for an isolated $\mathrm{As}_{\mathrm{Ga}}$ antisite, which we have calculated using the same approximations.

The total energy curves in Fig. 3 predict the existence of the metastability, but due to the omission of full ionic relaxations these curves cannot be used to obtain the barrier between the stable and the metastable configurations. A 32atom-site supercell has been employed in the calculations of the total energies presented in Fig. 3. In the following we present results of the calculations with a 64-atom-site supercell and with all the atoms allowed to move without any symmetry constraints to the (local) minimum energy configuration. For example, we have calculated the atomic relaxations for each energy minimum shown in Fig. 3. The displaced arsenic ion stays in the metastable interstitial configuration for all charge states, proving the existence of an energy barrier. The calculated total-energy difference be- 
tween the substitutional and the metastable interstitial states is between 0.24 and $0.95 \mathrm{eV}$, depending on the charge state of the defect. The energy difference is smallest for the 2charge state and highest for the neutral charge state.

In the substitutional configuration of the antistructure defect the interaction between the two antisites is rather weak. The distance between the two antisites is too large for a direct covalent bonding and thus the interaction is mainly Coulombic. With respect to the isolated $\mathrm{As}_{\mathrm{Ga}}$ antisite the main effect of the $\mathrm{Ga}_{\mathrm{As}}$ antisite in the defect complex is to change the total charge state of the complex, but the ionization levels (see Fig. 2) and also the other properties of the substitutional $\mathrm{As}_{\mathrm{Ga}}$ antisite do not differ significantly from those of the isolated antisite. The $\mathrm{As}_{\mathrm{Ga}}$ antisite-derived ionization levels are slightly lower in the case of the antistructure defect, which can also be regarded as a Stark effect due to the nearby $\mathrm{Ga}_{\mathrm{As}}$ antisite.

In the case of the chosen [111] direction for the movement of the $\mathrm{As}_{\mathrm{Ga}}$ antisite, the distance between the two antisites in the metastable interstitial configuration is $\sim 30 \%$ less than in the substitutional configuration. Thus the interaction between $V_{\mathrm{Ga}} \mathrm{As}_{i}$ and $\mathrm{Ga}_{\mathrm{As}}$ in the metastable configuration is much stronger and the properties of the $V_{\mathrm{Ga}} \mathrm{As}_{i}$ in a pair differ to a great extent from an isolated $V_{\mathrm{Ga}} \mathrm{As}_{i}$. The fact that one As ion is substituted around the metastable position of the $\mathrm{As}_{i}$ by a $\mathrm{Ga}$ ion with a smaller valence charge pushes the $\mathrm{V}_{\mathrm{Ga}} \mathrm{As}_{i}$-derived deep level higher in energy. This makes its partial occupancy or even its emptying possible and introduces ionization levels into the band gap. Moreover, the interaction pulls a state from the conduction band to the band gap and a triply negative charge state is possible for the antistructure defect in the metastable configuration.

Our calculations show that the $\left(V_{\mathrm{Ga}} \mathrm{As}_{i}\right)^{0}$ defect arising from an isolated $\mathrm{As}_{\mathrm{Ga}}$ antisite does not have ionization levels in the band gap in its metastable configuration. This is because there are no empty deep levels available in the band gap and the emptying of the occupied deep level by one electron leads to the lowering of the level into the valence band and its refilling. The lack of the ionization levels means that light absorption for this defect is very low. According to the experiments for the EL2 defect an applied pressure brings an ionization level from the conduction band to the band gap, resulting in strong light absorption. ${ }^{19}$ The ionized defect may capture a photocreated hole so that an excited neutral EL2 defect in the metastable state results. ${ }^{19}$ In this state there is no energy barrier towards the substitutional configuration and therefore the pressure enables a photorecovery process. This pressure behavior has been theoretically predicted also for the isolated $\mathrm{As}_{\mathrm{Ga}}$ antisite. ${ }^{20}$ In the case of the metastable configuration of the antistructure defect $\left(V_{\mathrm{Ga}} \mathrm{As}_{i} \mathrm{Ga}_{\mathrm{As}}\right)$ there are, due to the interaction between the $\mathrm{V}_{\mathrm{Ga}} \mathrm{As}_{i^{-}}$and $\mathrm{Ga}_{\mathrm{As}}$-derived states, ionization levels in the band gap already without applied pressure. The positions of these levels in the band gap are given in Fig 2. The ionization levels cause the defect to absorb light strongly.

The illumination of the antistructure defect by $1.1 \mathrm{eV}$ light triggers the transition to the metastable state. ${ }^{6,9}$ This is analogous to the EL2 defect. However, an important difference between the EL2 and the antistructure pairs is that the latter cannot be excited to the metastable state using unfiltered white light. ${ }^{6}$ The optical properties of the anti-structure

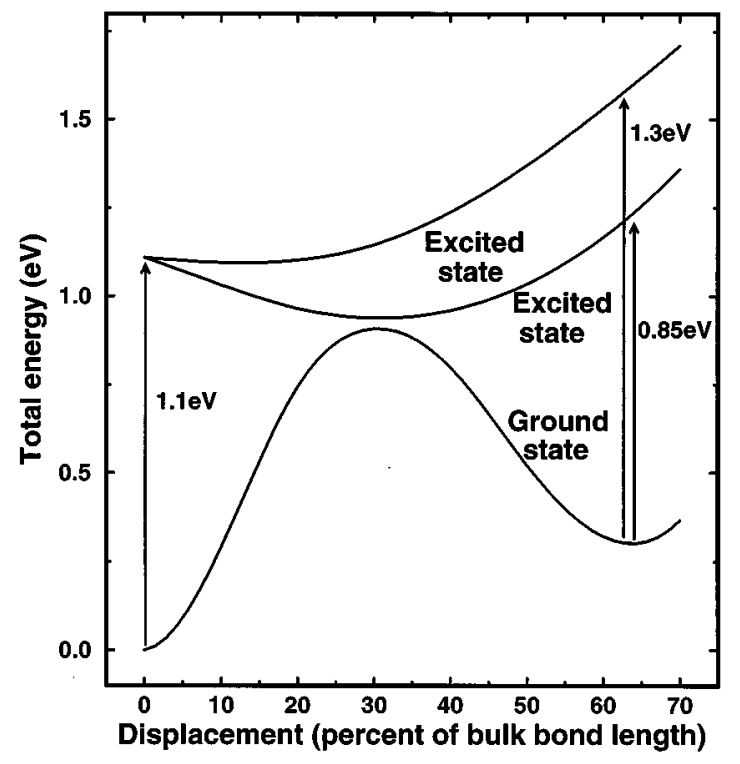

FIG. 4. Schematic model of possible optical excitation processes for the antistructure defect. The experimental values (Ref. 9) for the photon energies are shown. The lowest unoccupied single-particle levels of the antistructure defect are degenerate in the substitutional configuration of the defect. In the interstitial configuration the lowest normally empty single-particle levels of the antistructure defect are nondegenerate. Two empty single-particle levels in the band gap explain the observed two possible photon energies for the photorecovery of the defect.

defect can be explained by the model given in Fig. 4. In the excited states one electron is shifted to a higher, originally unoccupied energy level, leaving a hole behind. For the stable substitutional configuration the only unoccupied electron level in the band gap is in practice degenerate in the case of $1-$ and $2-$ charge states. Thus the excitation energy is unique. The degeneracy of these levels is due to the symmetry; these states correspond to the degenerate $e$ state of the $\mathrm{As}_{\mathrm{Ga}}$. The symmetry lowering due to the $\mathrm{Ga}_{\mathrm{As}}$ in the nextnearest-neighbor position is not strong enough to lift the degeneracy. Then, in the resulting excited state the total energy is lowered by the Jahn-Teller effect when the $\mathrm{As}_{\mathrm{Ga}}$ antisite is displaced in the [111] direction. This causes the shift to the metastable state similarly to the LLR model for the EL2 defect. ${ }^{17}$ For the antistructure defect in the metastable interstitial configuration this level is split into two in the band gap and there are two possible excited states. The total energy of these states is lowered when the $\mathrm{As}_{\mathrm{Ga}}$ antisite moves back towards to the substitutional configuration, resulting in the recovery to the stable state.

We have calculated estimates for the photon energies needed to excite an electron from the highest occupied defect levels to the lowest unoccupied ones. The single-particle energy differences give for the substitutional configuration the photon energy of $1.0 \mathrm{eV}$ and for the metastable configuration two energies of 0.6 and $1.0 \mathrm{eV} .^{21}$ These energies correspond well to the experimental energies shown in Fig. 4. Thus the model explains all the processes activated by monochromatic light.

The antistructure gallium antisite is in a diamagnetic state 
$\left(\mathrm{Ga}_{\mathrm{As}}{ }^{2-}\right)$, in agreement with experiments ${ }^{7}$ for the stable state of the antistructure defect. This assignment of diamagnetism for $\mathrm{Ga}_{\mathrm{As}}$ is valid for all charge states of the anti-structure defect. Our calculations also predict, in accordance with the experiments, ${ }^{7}$ the existence of the paramagnetic charge state $(1-)$ for the antistructure defect in both the stable and metastable states when the Fermi level is near the midgap.

In conclusion, we have made calculations for the nextnearest-neighbor antistructure pair in GaAs. The calculated properties are in excellent agreement with experimental results for electron-irradiated samples. ${ }^{6-9}$ The observed metastability is connected to the large lattice relaxation of the arsenic antisite. A gallium vacancy is created in the complex during the transition to the metastable interstitial configuration. The main difference between the isolated arsenic antisite and the antistructure defect is that the isolated antisite does not have any ionization levels in the band gap in the metastable state, whereas the antistructure defect has ionization levels in the gap both in the stable and in the metastable state. Furthermore, the optical recovery of the antistructure defect from the metastable state is possible at two photon energies. The optical recovery of the anti-structure defect also explains the inefficiency of the illumination by unfiltered white light to excite the defect from the stable state to the metastable one. Our results confirm the microscopic next-nearest-neighbor pair as the observed metastable defect induced by electron irradiation.

The authors wish to thank Dr. K. Saarinen and Professor P. Hautojärvi for many valuable discussions. We also acknowledge the generous computing resources from the Center for Scientific Computing (CSC). This research has been supported by the Academy of Finland through a MATRA grant.
${ }^{1}$ M. Kaminska and E. R. Weber, in Imperfections in III/V Materials, Semiconductors and Semimetals Vol. 38, edited by E. R. Weber (Academic Press, New York, 1993).

${ }^{2}$ See, for example, U. Scherz and M. Scheffler, in Imperfections in $I I I / V$ Materials (Ref. 1), pp. 2-58, and references therein.

${ }^{3}$ A. Pillukat and P. Ehrhart, Appl. Phys. Lett. 60, 2794 (1992); K. Krambrock, J.-M. Spaeth, C. Delerue, G. Allan, and M. Lannoo, Phys. Rev. B 45, 1481 (1992); E. Christoffel, A. Goltzene, and G. Schwab, J. Appl. Phys. 66, 5648 (1989); M. O. Manasreh and D. W. Fisher, Appl. Phys. Lett. 53, 2429 (1988).

${ }^{4}$ A. Goltzene, B. Meyer, and C. Schwab, Appl. Phys. Lett. 54, 907 (1989); M. O. Manasreh, D. W. Fischer, and B. C. Covington, Phys. Rev. B 37, 6567 (1988).

${ }^{5}$ P. Omling, E. R. Weber, and L. Samuelson, Phys. Rev. B 33, 5880 (1986); M. Hoinkis and E. R. Weber, ibid. 40, 3872 (1989); J. Krüger and H. Alexander, Mater. Sci. Forum 83-87, 899 (1992).

${ }^{6}$ M. Hesse, F. K. Koschnick, K. Krambrock, and J.-M. Spaeth, Solid State Commun. 92, 207 (1994).

${ }^{7}$ K. Krambrock and J.-M. Spaeth, Phys. Rev. B 47, 3987 (1993).

${ }^{8}$ K. Saarinen, S. Kuisma, J. Mäkinen, P. Hautojärvi, M. Törnqvist, and C. Corbel, Phys. Rev. B 51, 14152 (1995).

${ }^{9}$ S. Kuisma, K. Saarinen, P. Hautojärvi, and C. Corbel, Phys. Rev. B 53, 7588 (1996).
${ }^{10}$ R. Car and M. Parrinello, Phys. Rev. Lett. 55, 2471 (1985).

${ }^{11}$ D. M. Ceperley and B. J. Alder, Phys. Rev. Lett. 45, 566 (1980); J. Perdew and A. Zunger, Phys. Rev. B 23, 5048 (1981).

${ }^{12}$ G. B. Bachelet, D. R. Hamann, and M. Schlüter, Phys. Rev. B 26, 4199 (1982); R. Stumpf, X. Gonze, and M. Scheffler (unpublished).

${ }^{13}$ H. J. Monkhorst and J. D. Pack, Phys. Rev. B 13, 5188 (1976).

${ }^{14}$ D. J. Chadi and M. L. Cohen, Phys. Rev. B 8, 5747 (1973).

${ }^{15}$ S. Pöykkö, M. J. Puska, M. Alatalo, and R. M. Nieminen, Phys. Rev. B 54, 7909 (1996).

${ }^{16}$ S. B. Zhang and J. E. Northrup, Phys. Rev. Lett. 67, 2339 (1991).

${ }^{17}$ J. Dabrowski and M. Scheffler, Phys. Rev. Lett. 60, 2183 (1988); D. J. Chadi and K. J. Chang, ibid. 61, 2187 (1988).

${ }^{18}$ T. Mattila and R. M. Nieminen, Phys. Rev. Lett. 74, 2721 (1995).

${ }^{19}$ M. Baj, P. Dreszer, and A. Babinski, Phys. Rev. B 43, 2070 (1991); P. Dreszer, M. Baj, and K. Korzeniewski, Mater. Sci. Forum 83-87, 875 (1992); T. W. Steiner, M. K. Nissen, S. M. Wilson, Y. Lacroix, and M. L. W. Thewalt, Phys. Rev. B 47, 1265 (1993).

${ }^{20}$ C. Ziegler, U. Scherz, and M. Scheffler, Phys. Rev. B 47, 16624 (1993).

${ }^{21}$ We estimate the photon energy by calculating the energy eigenvalue difference between the corresponding Kohn-Sham eigenstates. 